\title{
NOTAS HISTÓRICAS DEL PRIMER SEISMO REGISTRADO EN LA CIUDAD DE PUNTA ARENAS Y EN LA REGIÓN DE MAGALLANES EN FEBRERO DE 1879
}

ALFREDO PALACIOS R.*

\section{RESUMEN}

Las siguientes páginas tienen como objetivo dar a conocer los relatos del primer terremoto que cuenta con registro escrito en Punta Arenas y en la región de Magallanes. El dar a conocer estos testimonios no solo permite apreciar la magnitud de aquel movimiento telúrico, sino que también contribuye a aclarar dudas con respecto a su ocurrencia, ayuda a mejorar la cronología sísmica de la región y, sobre todo, proporciona la posibilidad de concientizar a la población a estar prevenida ante esta amenaza natural.

PALABRAS CLAVE: Punta Arenas, Magallanes, riesgos naturales, terremotos, sismicidad histórica.

\section{HISTORICAL NOTES FOR FIRST RECORDED EARTHQUAKE IN THE CITY OF PUNTA ARENAS AND THE MAGALLANES REGION IN FEBRUARY 1879}

\begin{abstract}
The following pages are intended to present the stories of the first earthquake that was recorded in Punta Arenas and the Magallanes region. The publiciz these testimonies not only allows you to appreciate the magnitude of the telluric movement, but it also helps to clarify doubts with respect to its occurrence, ing of helping to improve the seismic chronology of the region and, above all, give the possibility of raising awareness among the population to be warned for this natural threat.
\end{abstract}

KEY WORDS: Punta Arenas, Magallanes, natural hazards, earthquakes, historical seismicity. 


\section{ANTECEDENTES}

Desde la ocupación y colonización de la región de Magallanes, en 1843, y antes con la presencia de numerosos exploradores y viajeros extranjeros, no encontramos ningún registro escrito sobre la ocurrencia de algún acontecimiento telúrico en aquella zona hasta el mes de febrero de 1879 (Martinic, 1988. Sin duda, y debido a la activa geodinámica que presentaba el resto de las regiones de Chile, esta singular "ausencia" llamó la atención de aquellos estudiosos que desde la década del cincuenta del siglo XIX comenzaron a realizar sus investigaciones geológicas y meteorológicas en la naciente ciudad de Punta Arenas ${ }^{1}$. A este respecto, en 1859 Ignacio Domeyko manifestó con asombro a las autoridades de la Universidad de Chile el hecho de que ningún científico haya sentido "un solo temblor” en aquellas latitudes (Domeyko, 1859. Por otra parte, si se considera que en aquella época algunos autores especulaban con el origen volcánico de los movimientos telúricos (Navarro, 1908, y se tienen en cuenta las historias y leyendas sobre los volcanes rugientes de la Tierra del Fuego (Pérez, 2012), resulta aún más llamativo ese silencio sísmico.

\section{EL TERREMOTO DEL 1 DE FEBRERO DE 1879}

Este proceso geológico, descrito en algunas cronologías y catálogos sísmicos como un "gran temblor" (Montessus de Ballore, 1912:193. Brüggen, 1943:123) o "terremoto débil" (Bustos, 1931: 66), se produjo en la madrugada del 1 de febrero de 1879. Algunas de las escasas publicaciones que dan cuenta de este evento telúrico señalan que en la población del territorio magallánico -que según el censo de 1875 ascendía a 1144 habitantes (Oficina Central de Estadísticas, 1877: 5)- no se registraron heridos ni daños materiales de consideración (Martinic, 1988: 30). Sin embargo, el espanto que provocó el movimiento llevó a que el gobernador de la colonia de Magallanes, en una medida que hoy denominaríamos de mitigación, prohibiese la construcción de edificaciones de ladrillo, ya que entendía que la región estaba muy expuesta a los riesgos sísmicos y que, además, la población

${ }^{1}$ Estas notas forman parte de una investigación mayor sobre los terremotos ocurridos en Chile entre los siglos XVI y XIX. debió su salvación a sus casas hechas exclusivamente de madera (Baeriswyl, 2001: 17). Por otra parte, algunos científicos indican que esta coyuntura se produjo como respuesta al movimiento transcurrente entre las placas Sudamericana, Scotia, y Antártica (Comte \& Barrientos, 1997) y otros investigadores añaden que aquel remezón alcanzó una magnitud de “7 a 7,5" (Lomnitz, 1971). Del mismo modo, estudios recientes identifican a la Falla de Magallanes, cerca del cabo Froward, como el probable epicentro de este singular terremoto (Cisternas \& Vera, 2008).

\section{Los testigos y sus relatos}

A continuación, y con la intención de complementar la escasa información referente a este fenómeno sísmico, reproduciremos los relatos de seis testigos contemporáneos a los hechos. El primero de estos corresponde al del antiguo gobernador de Magallanes, Diego Dublé Almeida, quien sintió el terremoto durante su paso por Punta Arenas. La segunda relación fue extraída del diario de viaje de Florence Dixie que experimentó el movimiento en la zona de Laguna Blanca, al noreste de Punta Arenas. La tercera narración es la de los oficiales de la corveta Magallanes, Juan M. Simpson y Federico Chaigneau, quienes estaban en un campamento próximo a la bahía Pinto, en la costa norte del mar de Skyring. El cuarto testimonio nos lo entrega el teniente Ramón Serrano quien se encontraba a medio camino entre la bahía de San Sebastián y el cabo del Espíritu Santo en Tierra del Fuego. El quinto relato viene de manos del explorador Juan Tomás Rogers desde el paraje de Malogro en Argentina. Y finalmente, el último texto lo redactó el reverendo Thomas Bridges desde la misión trasandina de Ushuaia.

El $1^{\circ}$. de Febrero, a las 3.40 a.m., hubo en Punta Arenas un fuerte temblor que duró más de un minuto y que merece el nombre de terremoto. No causó daño en ninguno de los edificios porque todos son de madera y de un piso. El espanto en la población fue extraordinario. Aquí jamás había temblado y todos temían algún cataclismo. Ha continuado temblando por tres días, pero los estremecimientos de la tierra no han sido tan fuertes como el primero. Los tripulantes de la "Magicienne", 
buque de guerra francés que se hallaba al ancla en Puerto Cumberland, en el Estrecho, sintieron el gran temblor. Todos a bordo creyeron que alguna explosión había ocurrido en la máquina del buque, fue tal el ruido que produjo (Dublé Almeida, 1938:279).

Un fuerte y retumbante ruido se escuchó en el aire $y$ antes de que tuviera tiempo de preguntarme que podía significar, un desplazamiento de la tierra parecido a una marejada me lanzó volando sobre mi espalda y como por arte de magia el silencioso campo volvió a la vida con gritos de temor y sorpresa mientras todos escapaban consternados de sus carpas. Las sacudidas siguieron una y otra vez pero cada vez más débiles y pasado cinco minutos habían cesado del todo. Pero pasó un rato antes de que recuperáramos la tranquilidad. Ésta era la primera vez que experimentaba un terremoto y tan enfermante sensación de desamparo que te asalta durante las sacudidas de la tierra serian -diría yodifíciles de igualar. Nuestros guías dijeron que nunca habian sentido un terremoto en la Patagonia anteriormente, ni habian tenido conocimiento de que hubiera habido alguno (Dixie, 1996:110).

Día 31. [...] A las 3.45 A.M., mientras estábamos entregados al reposo, fuimos recordados por un fuerte temblor de tierra cuya duración fue de $1^{\mathrm{m}}$. 30 . La oscilación tuvo lugar de N. a S., no sintiéndose ruido sino al final del primer remezón. Siguieron 2 más, pero de muy poca intensidad y con intervalos de 4 minutos el primero. Los árboles se movian como impulsados por un huracán, quedando largo rato oscilando en la dirección antedicha. La mañana estaba en calma, asi es que pudimos percibir los menores detalles de este fenómeno, que pocas veces hemos sentido tan fuerte y de tan larga duración, pues la tierra quedó vibrando por algún tiempo sin poder calcular la dirección del movimiento, que solo fue apreciable en la primera sacudida
(Simpson \& Chaigneau, 1880: 83).

Febrero $1^{\circ}$. A las 3.50 A.M., tiempo verdadero, se sintió un prolongado temblor de tierra de fuerza extraordinaria, tanto que me temo haya sido un terremoto en alguna parte. Su fuerza fue tanta que a pesar de estar ensacado, ya que no encamado, y en el suelo, me sacudió con tanta violencia que me hizo deslizarme como 2 decímetros sobre el terreno que era algo inclinado. Fue acompañado de un fuerte ruido y puedo decir que es el más fuerte que he sentido en mi vida. Las oscilaciones parecian venir del S. y su duración la estimo en más de 1 minuto. Jamás he oído hablar de temblores ni recuerdo haberlos sentido en Punta Arenas, $y$ hoy he preguntado a dos de los hombres que me acompañaban, los que han residido por más de diez años en la colonia; y ellos me aseguraron no haber sentido ni oído hablar de temblores ahi (Serrano, 1880:188-189).

Febrero $1^{\circ}$. A las 3.30 A. M. de este día se sintió un largo y fuerte remezón de tierra, fenómeno muy raro en la parte austral del continente sud-americano; pues estábamos informados de que en Punta Arenas no se había dejado sentir ningún temblor de tierra desde la existencia de la colonia. (Rogers, 1880:116).

El $1^{\circ}$ de febrero a las cinco, tuvimos una sucesión de remezones de terremoto, lo bastante fuerte para despertar a casi todos y hacer que caminar fuera algo difícil. Se derramó la leche de las vasijas y se sintió en toda la región. Fred lo sintió en la Bahía Gretton (Bridges, 2001:104).

De esta manera, y gracias al conjunto y simultaneidad de estos relatos, se puede llegar a determinar el impacto y la extensión geográfica del terremoto (Fig. 1), la cual representa, según el análisis del profesor Martinic un rango longitudinal de no menos de 600 kilómetros y latitudinal de unos 400 kilómetros (Martinic, 2008: 14). 


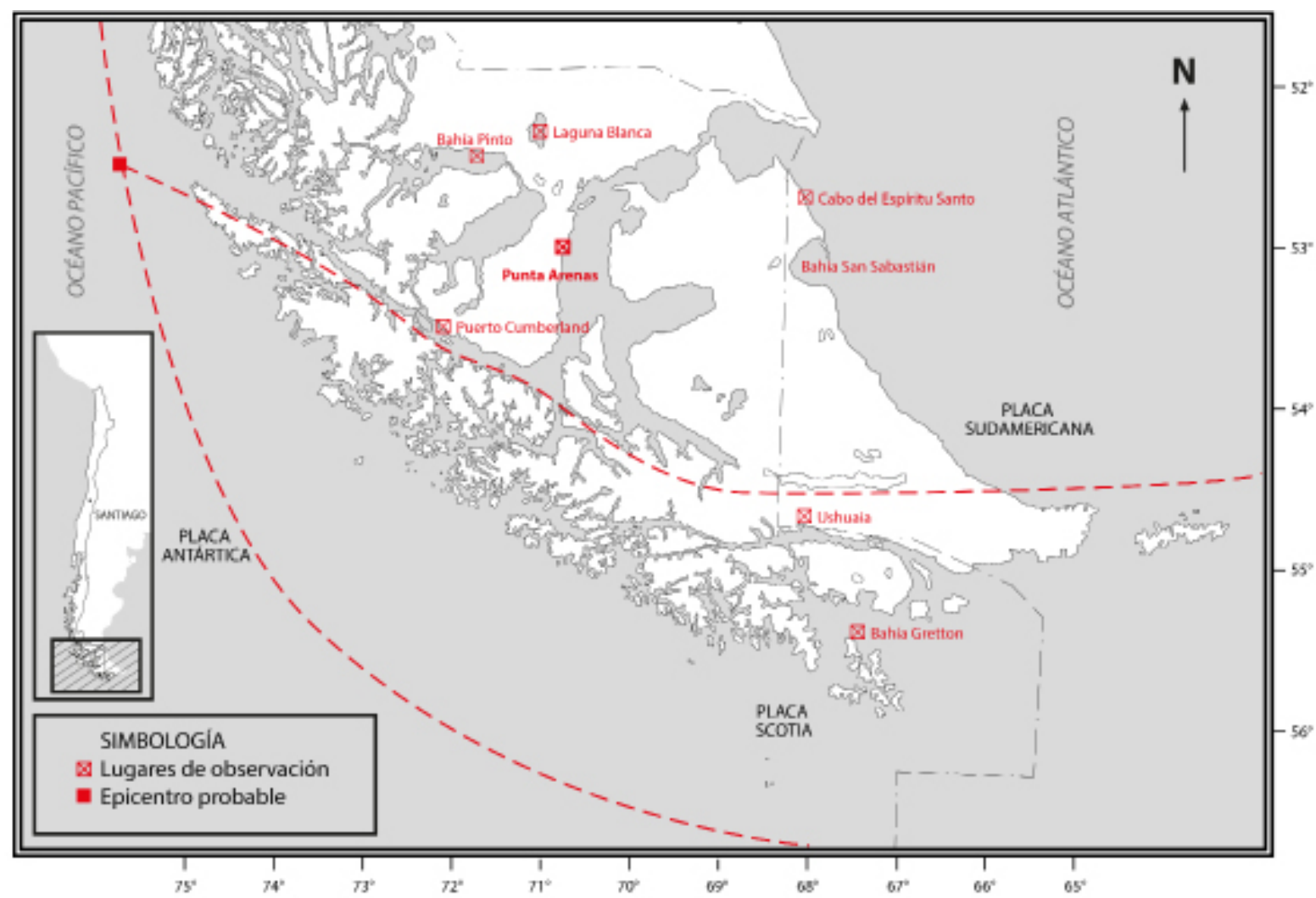

FUENTE: ELABORACIÓN PAOPIA

Fig. 1 Mapa de localización de lugares con observación del sismo de 1879 y su probable epicentro.

\section{BIBLIOGRAFIA}

Baeriswyl, D. (2001). Arquitectura en Punta Arenas, primeras edificaciones en ladrillos: 1892-1935. Punta Arenas: Talleres de la Prensa Austral.

Bridges, T. (2001). Los indios del último confín. Buenos Aires: Zagier \& Urruty Publications.

Brüggen, J. (1943). Contribución a la geología sísmica de Chile. Revista Chilena de Historia y Geografía, 103, 108-174.

Bustos, J. (1931). Estudio sismológico de Chile con los temblores y terremotos producidos en los últimos cuatro siglos. Anales de la Universidad de Chile, 1(1), 59-91.

Cisternas, A., \& Vera, E. (2008). Sismos históricos y recientes en Magallanes. Magallania, 36(1), 43-51.

Comte, D., \& Barrientos, S. (1997). Potencial sísmico en Chile. Actas del VIII Congreso Geológico Chileno, III, 1755-1759.

Dixie, F. (1996). A través de la Patagonia. Punta Arenas: Ediciones de la Universidad de Magallanes.
Domeyko, I. (1859). Estudios geográficos sobre Chile. Anales de la Universidad de Chile, XVI, 18-61.

Dublé Almeida, D. (1938). Diario de viaje al río Santa Cruz, Patagonia. Revista Chilena de Historia y Geografía, 93, 254-279.

Lomnitz, C. (1971). Grandes terremotos y tsunamis en Chile durante el periodo 1535-1955. Revista Geofísica Panamericana, 1(1), 151-175.

Martinic, M. (1988). El gran temblor de tierra de 1879 en la Patagonia austral. Revista Patagónica, 37,30-31.

Martinic, M. (2008). Registro histórico de antecedentes volcánicos y sísmicos en la Patagonia Austral y la Tierra del Fuego. Magallania, 36(2), 5-18.

Montessus de Ballore, F. (1912). Geografía sísmica de Chile. Revista Chilena de Historia y Geografía, 7, 178-195.

Navarro, L. (1908). Censo general de población y edificación, industria, ganadería y minería del territorio de Magallanes República de Chile. Punta Arenas: Talleres de la Imprenta de El Magallanes. 
Oficina Central de Estadística (1877). Quinto censo general de la población de Chile. Santiago: Imprenta Nacional.

Pérez, V. (2012). Jorge Cristian Schythe: El gobernador (1853-1858) y meteorólogo de Punta Arenas, Región de Magallanes. Anales del Instituto de la Patagonia, 40(1), 169-172.

Rogers, J. T. (1880). Segunda exploración de la parte austral de la Patagonia. Anuario Hidrográfico de la Marina de Chile, VI, 97-150.

Serrano, R. (1880). Diario de la excursión a la isla grande de la Tierra del Fuego durante los meses de enero y febrero de 1879. Anuario Hidrográfico de la Marina de Chile, VI, 151-204.

Simpson, J. \& F. Chaigneau. (1880). Diario llevado por los tenientes Simpson y Chaigneau. Anuario Hidrográfico de la Marina de Chile, VI, 73-96. 
